DRUG RESISTANCE

\section{Sensitive to change}

The Fanconi anaemia (FANC)BRCA1 pathway is required for DNA repair in response to damage caused by DNA crosslinking agents, such as cisplatin. Patients with ovarian cancer are given cisplatin as part of their standard therapy, but they often become resistant to this drug. The molecular mechanisms involved in cisplatin sensitivity and acquired resistance are unknown, so Taniguchi et al. investigated the role of the FANC-BRCA1 pathway in this process. The authors report in Nature Medicine that resistance of ovarian cancer cell lines to cisplatin depends on the methylation status of one of the key FANC genes, FANCF.

Five of the FANC proteins (A, C, E, $\mathrm{F}$ and $\mathrm{G}$ ) are subunits of a nuclear complex that is required for monoubiquitylation of the FANCD2 protein, which interacts with BRCA1 to initiate repair. Two ovarian cancer cell lines that are hypersensitive to cisplatin do not express the mono-ubiquitylated form of FANCD2, but when the authors overexpressed FANCF - but not other FANC proteins - ubiquitylation of FANCD2, and its functionality, was restored. Consequently, the corrected cells were more resistant to cisplatin than the original cell line.

FANCF was not mutated in the cisplatin-sensitive cells, but the gene contains a CpG island, which might inactivate it when methylated. The authors treated these cells with a demethylating agent -5 -aza-2'deoxycytidine - which increased FANCF mRNA and protein expres-

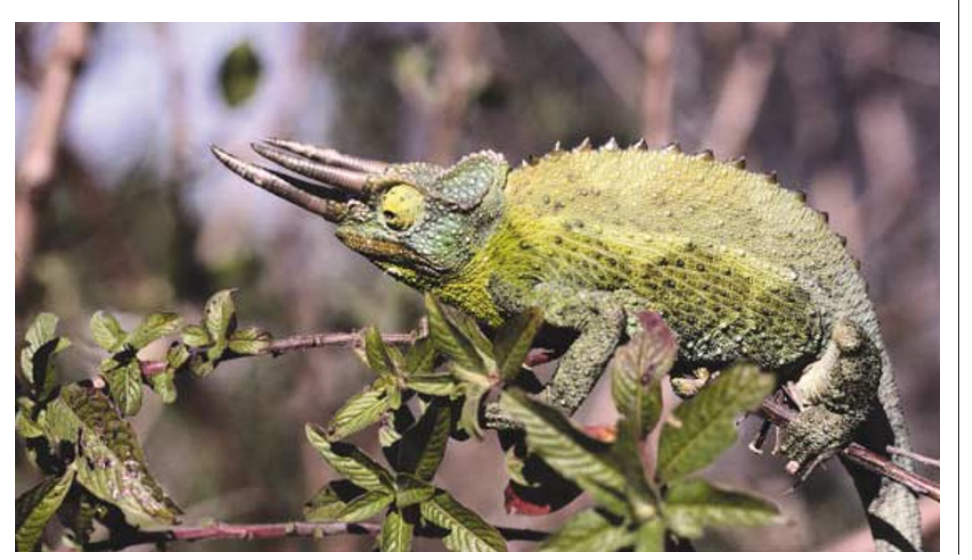

sion and partially restored FANCD2 mono-ubiquitylation. Methylationspecific polymerase chain reaction confirmed that $F A N C F$ is methylated in the cisplatin-sensitive cell lines, but not in normal ovarian cells.

So, FANCF methylation can regulate cisplatin sensitivity, but does demethylation correlate with resistance? A cisplatin-resistant derivative of a cisplatin-sensitive ovarian cancer cell line expressed low levels of FANCF. Experimental overexpression of FANCF did not greatly increase the resistance of these cells, but sequencing of the $\mathrm{CpG}$ island showed that FANCF was demethylated in the resistant cells. is methylated and thereby inactivated early in ovarian cancer progression, and exposure to cisplatin at this stage leads to cell kill. However, in some tumour cells, FANCF then becomes demethylated, resulting in outgrowth of resistant cells. In the future, development of a targeted inhibitor of ubiquitylation of FANCD2 could bypass the influence of FANCF and be used to resensitize tumours in patients who have become resistant to cisplatin.

Ezzie Hutchinson

\section{(2) References and links} ORIGINAL RESEARCH PAPER Taniguchi, T. et al. Disruption of the Fanconi anemia-BRCA et al. Dissuption of the Fanconi anemia-BRCA
pathway in cisplatin-sensitive ovarian tumors. Nature Med. 7 Apr 2003 (doi: 10.1038/nm852) WEB SITE

Alan D. D'Andrea's lab:

http://www dfci hanardedu/pat/dana-

farber/detail.asp? personID=43\&RD=True
The authors suggest that FANCF

\section{TRIAL WATCH}

\section{Detective work}

The National Cancer Institute's Early Detection Research Network (EDRN) is launching its first Phase III study of a cancer biomarker. The study is designed to evaluate microsatellite instability as a marker for recurrent bladder cancer.

The trial will test a microsatellite instability assay developed by David Sidransky and colleagues. This assay measures changes in about 20 microsatellite loci in cells sloughed off the bladder wall and into the urine. The study, expected to take 3 years to complete, will enroll 300 patients with bladder cancer. It will compare microsatellite analysis with bladder endoscopy and cytology — the current standards for monitoring bladder cancer.

Screening for bladder cancer is challenging, because of its relatively low prevalence. But if this approach proves successful, similar microsatellite instability tests might be developed to screen for other cancers that shed cells into body fluids, such as oral and gastrointestinal tumours.

This multicentre collaboration is the first of many planned for the EDRN's broad consortium of biomarker discovery and validation laboratories.

FURTHER INFORMATION $\mathrm{http}: / /$ www3.cancer.gov/prevention/cbrg/edrn/

\section{HRT patches for men}

Results of a pilot study to assess the impact of transdermal oestradiol therapy on advanced prostate cancer in men are reported in the May issue of the Journal of Urology.

Androgen suppression using hormones or surgery is the standard treatment for patients with advanced prostate cancer. This approach, however, has many side effects, including symptoms of andropause and osteoporosis. Clinical trials of oral oestrogens, which lower testosterone levels, were abandoned because of cardiovascular toxicity.

Ockrim et al. tested the effects of oestrogen patches in patients with prostate cancer. They found that in patients who wore two or more patches a week, oestradiol levels increased to more than $1,000 \mathrm{pmol} / \mathrm{L}$. The concentrations of testosterone subsequently fell to castrate levels within 3 weeks in all patients. Side effects seen with oral oestrogens - such as thrombolic activation and impeded vascular flow - were not reported, and only one patient had a cardiovascular complication (fluid retention). The toxic effects seen with other androgensuppression therapies were also avoided - bone mineral density actually increased and no patients had hot flushes. A total of $80 \%$ of men did have mild or moderate distress due to enlargement of breasts.

In addition, all patients showed an average reduction in prostate-specific antigen (PSA) concentrations of $95.1 \%$ — PSA is a surrogate marker of disease regression. The authors point out that transdermal oestradiol patches are one-tenth of the cost of current therapy for advanced prostate cancer and, together with the preliminary indications that the patches are effective and cause few side effects, this makes them an attractive option for further investigation.

ORIGINAL RESEARCH PAPER Ockrim, J. L. et al. Transdermal estradiol therapy for advanced prostate cancer -forward to the past. J. Urol. 169, 1735-1737 (2003) 